\title{
A NEW INTEGRAL EXPRESSION FOR THE BERNOULLI NUMBERS AND EXPLICIT FORMULAS
}

\section{A PREPRINT}

\section{Sumit Kumar Jha}

International Institute of Information Technology

Hyderabad, India

kumarjha.sumit@research.iiit.ac.in

June 13, 2019

\begin{abstract}
In this brief note, we give, a possibly new, integral expression for the Bernoulli numbers involving the polylogarithm function. We use this to derive two explicit formulas for them in terms of the Stirling numbers of the second kind, and the Eulerian numbers.
\end{abstract}

Keywords Bernoulli numbers · Stirling numbers of the second kind $\cdot$ Eulerian numbers $\cdot$ Polylogarithm function AMS Classification: 11B68

Definition 1. The Bernoulli numbers $B_{n}$ can be defined by the following generating function

$$
\frac{t}{e^{t}-1}=\sum_{n \geq 0} \frac{B_{n} t^{n}}{n !}
$$

where $|t|<2 \pi$.

Definition 2. The polylogarithm function $\mathbf{L i}_{s}(z)$ is defined by the following power series in $z$

$$
\mathbf{L i}_{s}(z)=\sum_{k=1}^{\infty} \frac{z^{k}}{k^{s}}
$$

where $|z|<1$.

When $s=-r$, a negative integer, then polylogarithms reduce to ratio of polynomials in $z$. First few values of which are

$$
\begin{gathered}
\mathrm{Li}_{-1}(z)=\frac{z}{(1-z)^{2}} \\
\mathrm{Li}_{-2}(z)=\frac{z(1+z)}{(1-z)^{3}} \\
\mathrm{Li}_{-2}(z)=\frac{z(1+z)}{(1-z)^{3}} \\
\operatorname{Li}_{-3}(z)=\frac{z\left(1+4 z+z^{2}\right)}{(1-z)^{4}} \\
\operatorname{Li}_{-4}(z)=\frac{z(1+z)\left(1+10 z+z^{2}\right)}{(1-z)^{5}} .
\end{gathered}
$$

We prove the following here

Theorem 1. We have

$$
B_{r}=\int_{0}^{\infty} \frac{\mathbf{L i}_{-r}(-x)}{1+x} d x
$$


Proof. We have, the following generating function [1],

$$
\frac{\mathbf{L i}_{-r}(-x)}{1+x}=\sum_{n=0}^{\infty} S_{r}(n)(-x)^{n}
$$

where $S_{r}(n)=\sum_{k=1}^{n} k^{r}$ for $n \geq 1$, and $S_{r}(0)=0$. Next, we use Ramanujan's Master Theorem (RMT) from [2], which is,

$$
\int_{0}^{\infty} x^{n-1}\left\{\phi(0)-x \phi(1)+x^{2} \phi(2)-\cdots\right\} d x=\frac{\pi}{\sin n \pi} \phi(-n)
$$

where the integral is convergent for $0<\boldsymbol{R e}(n)<1$, and after certain conditions are satisfied by $\phi$. Using RMT with equation (4) gives us

$$
\lim _{n \rightarrow 1} \int_{0}^{\infty} x^{n-1} \frac{\mathbf{L i}_{-r}(-x)}{1+x} d x=\lim _{n \rightarrow 1} \frac{\pi}{\sin n \pi}\left(\frac{B_{r+1}(1-n)-B_{r+1}}{r+1}\right)=B_{r} .
$$

As a consequence of the above result, we can obtain

Corollary 1. We have

and

$$
B_{r}=\sum_{k=1}^{r}(-1)^{k} \cdot k ! \frac{S(r, k)}{k+1},
$$

$$
(r+1) B_{r}=\sum_{l=1}^{r}(-1)^{l}\left\langle\begin{array}{c}
r \\
r-l
\end{array}\right\rangle\left(\begin{array}{l}
r \\
l
\end{array}\right)^{-1} .
$$

Here $S(r, k)$, and $\left\langle\begin{array}{r}r \\ r-l\end{array}\right\rangle$ represent the Stirling numbers of the second kind, and the Eulerian numbers respectively.

Proof. To prove (7), we use the following representation from note [3]

$$
\mathbf{L i}_{-r}(-x)=\sum_{k=1}^{r} k ! S(r, k)\left(\frac{1}{1+x}\right)^{k+1}(-x)^{k},
$$

which can be easily proved using induction on $r$.

Now, we deduce equation (7) from the following steps

$$
\begin{array}{rl}
B_{r}=\int_{0}^{\infty} \frac{\mathbf{L} \mathbf{i}_{-r}(-x)}{1+x} d x=\sum_{k=1}^{r}(-1)^{k} & k ! S(r, k) \int_{0}^{\infty} \frac{x^{k}}{(1+x)^{k+2}} d x \\
= & \sum_{k=1}^{r}(-1)^{k} \cdot k ! S(r, k) \frac{1}{k+1} .
\end{array}
$$

To prove (8), we use the following representation from [4]

$$
\mathbf{L i}_{-r}(-x)=\frac{1}{(1+x)^{r+1}} \cdot \sum_{j=0}^{r-1}\left\langle\begin{array}{l}
r \\
j
\end{array}\right\rangle \cdot(-x)^{r-j} .
$$

Now, to derive equation (8) we follow the steps below

$$
\begin{array}{r}
B_{r}=\int_{0}^{\infty} \frac{\mathbf{L i}_{-r}(-x)}{1+x} d x \\
=\sum_{l=1}^{r}(-1)^{l}\left\langle\begin{array}{c}
r \\
r-l
\end{array}\right\rangle \int_{0}^{\infty} \frac{x^{l}}{(1+x)^{r+2}} d x \\
=\sum_{l=1}^{r}(-1)^{l}\left\langle\begin{array}{c}
r \\
r-l
\end{array}\right\rangle \int_{0}^{\infty} \frac{x^{l}}{(1+x)^{r+2}} d x \\
=\sum_{l=1}^{r}(-1)^{l}\left\langle\begin{array}{c}
r \\
r-l
\end{array}\right\rangle \frac{\Gamma(l+1) \Gamma(r-l+1)}{\Gamma(r+2)} d x=\sum_{l=1}^{r}(-1)^{l}\left\langle\begin{array}{c}
r \\
r-l
\end{array}\right\rangle \frac{1}{r+1}\left(\begin{array}{c}
r \\
l
\end{array}\right)^{-1} .
\end{array}
$$

Here, $\Gamma(\cdot)$ is the Gamma function.

Remark 1. Two similar integral representations, and resulting explicit formulas appear in the note [5]. 


\section{References}

1. Weisstein, Eric W. "Polylogarithm." From MathWorld-A Wolfram Web Resource.

2. Amdeberhan, T., Espinosa, O., Gonzalez, I., Harrison, M., Moll, V. H., \& Straub, A. (2012). Ramanujan's master theorem. The Ramanujan Journal, 29(1-3), 103-120.

3. Stirling Numbers and Polylogarithms by Steven E. Landsburg. Link: http://www.landsburg.com/query.pdf

4. Weisstein, Eric W. "Eulerian Number." From MathWorld-A Wolfram Web Resource.

5. Jha, S.K., 2019. Two new explicit formulas for the Bernoulli Numbers. arXiv preprint arXiv:1905.11216. 